\title{
REVIEW ON RELATIONSHIP BETWEEN THE UNIVERSALITY CLASS OF THE KARDAR-PARISI-ZHANG EQUATION AND THE BALLISTIC DEPOSITION MODEL
}

\author{
Okhunjon SAYFIDINOV* and Gabriella BOGNAR \\ Miskolc-Egyetemvaros, 3515, Institute of Machine and Product Design \\ University of Miskolc, HUNGARY \\ E-mail: sayfidinov.ok@gmail.com
}

\begin{abstract}
We have analysed the research findings on the universality class and discussed the connection between the Kardar-Parisi-Zhang (KPZ) universality class and the ballistic deposition model in microscopic rules. In one dimension and 1+1 dimensions deviations are not important in the presence of noise. At the same time, they are very relevant for higher dimensions or deterministic evolution. Mostly, in the analyses a correction scale higher than 1280 has not been studied yet. Therefore, the growth of the interface for finite system size $\beta \geq 0.30$ value predicted by the KPZ universality class is still predominant. Also, values of $\alpha \geq 0.40, \beta \geq 0.30$, and $z \geq 1.16$ obtained from literature are consistent with the expected $\mathrm{KPZ}$ values of $\alpha=1 / 2, \beta=1 / 3$, and $z=3 / 2$. A connection between the ballistic deposition and the KPZ equation through the limiting procedure and by applying the perturbation method was also presented.
\end{abstract}

Keywords: universality class, ballistic deposition model, KPZ equation, finite-size scaling, perturbation method.

\section{Introduction}

One of the striking and the least investigated concepts is the universality class, which is one of the complex random systems. This system plays a main role in research on probability, in mathematical physics and statistical mechanics. In this article we will describe how various research findings have been presented, as well as some numerical analysis on the differences between ballistic deposition models and the KardarParisi-Zhang universality class. They lie in physical systems and mathematical models, including randomly growing interfaces, certain stochastic PDEs in random environments. Random matrices all demonstrate the same universal statistical behaviours in their long-time / large scale limit.

Over many years the study of surface growth of materials has been a complex area of research. As growth includes a number of disciplines such as metallurgy (solidification of alloys) [1], microelectronics (growth of nano devices) [2], biophysics (growth of proteins, cells and tumours, and cytoskeleton polymerization in the immune system) [3]. Each of them presents various problems, apparently with different underlying mechanisms. Therefore, the nature of crystalline is important for surface dynamics. In the microscale, crystalline surfaces exhibit two different structures: a rough surface (i) and a smooth or atomically flat surface (ii). Most metals and several organic components are included in the first category where their melting temperature is reached [4]. In this process, the surface fluctuates strongly and the concept of a crystalline plane is difficult to define. In the second category, surface atoms or molecules fit perfectly in a smooth atomically flat plane. For example, semiconductors, some metals, and more organic materials fall into this category.

The microscopic nature of the surface plays a major role in the physics of crystalline growth. Since many of unwanted bonds are present, the addition of new particles to the growing solid is quick for solids with rough surfaces. On the other hand, for a solid with a smooth or atomically flat surface adhesive particles are rare and the process is not easy. Growth on the surface can be two-dimensional (2D) caused adhesion of the

\footnotetext{
${ }^{*}$ To whom correspondence should be addressed
} 
atoms to the existing surface layer. This can occur when cutting a material that has a poor angle relative to the tightly joined plane. It results in a vicinal surface and results in screw dislocations. This category is growth problems driven by kinetics. The mother phase is classified into three prototypes: (i) growth from a solution (this case includes many organic materials, minerals, biological materials, etc.). In this solution, the elementary building blocks diffuse in solution [5] along the surface of the growing material and perform different kinetics, as mentioned above (e.g., nucleation, attachment to pre-existing steps, etc.); (ii) growth from a vapour; and (iii) growth from a beam [6, 7, 8]. In cases (ii) and (iii), the transport process in the mother phase is not relevant. The latter two categories are ballistic growth or molecular beam epitaxy (MBE) [9].

Initially, Vold [10] in 1959 used the term of ballistic deposition (or sticky block) model term, which mean a real growing interfaces and spatial correlation. The process can be represented by blocks. Here, a block will stick to the first edge against which it becomes incident. This process is illustrated in Figure 1(a, b). It creates overhanging blocks and defines the height function $h(t, x)$ as the maximum height above $x$ occupied by a box. Here, we are faced with the question of how this microscopic change manifests itself over time? In this deposition model, blocks appear to increase radically and the value of rate is unknown.
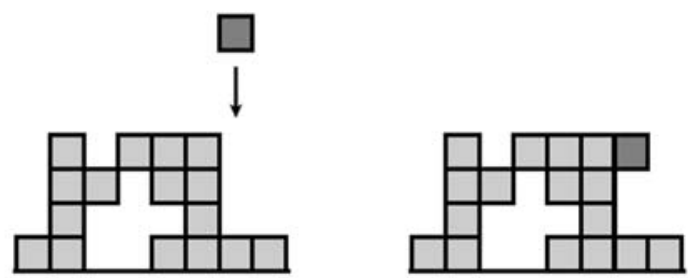

Fig.1(a). The ballistic deposition model.

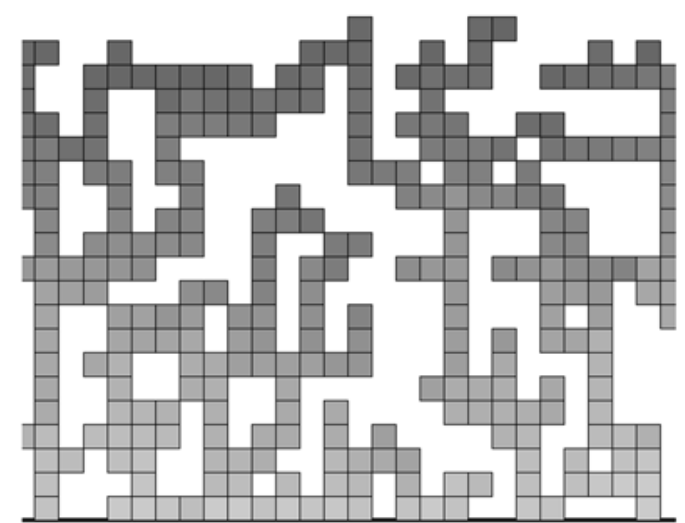

Fig.1(b). Result of ballistic deposition models.

Figure 1(a) illustrate the ballistic deposition model. In Fig.1(a) blocks fall from above each site with independent exponentially distributed waiting times. During falling they stick to the first edge to which they become incident. Figure 1(b) shows the result of ballistic deposition models driven by the same process of falling blocks. The ballistic model grows much faster and has a smoother more spatially correlated top interface than other models [1].

The longer time results are displayed in Fig.2., which also shows that the scale of fluctuations of $u(t, x)$ and the height function remain correlated transversally over a long distance. There are exact conjectures for these fluctuations. They are supposed to grow like $t^{1 / 3}$ and demonstrate a non-trivial correlation structure in a transversal scale of $t^{2 / 3}$ [11]. Precise predictions exist to provide the limiting distributions. Up to certain constants $c_{1}, c_{2}$, the sequence of scaled heights

$$
c_{2} t^{-\frac{1}{3}}\left(u(t, 0)-c_{1} t\right)
$$

should converge as Gaussian Orthogonal Ensemble (GOE) Tracy-Widom random variable. The Tracy-Widom distributions are illustrated as present-time bell curves and are named GOE or GUE (Gaussian Unitary Ensemble) derived from the random matrix ensembles in which these distributions were first observed by Tracy-Widom [12, 13].

It seems that ballistic deposition does not integrate with a probabilistic system. Obviously, there is a question how this prediction appeared? It came from an analysis of some similar growth processes that happen 
to be integrable. Ballistic deposition has similar characteristics to these models that are believed to be the key to KPZ class membership [11]:

- Locality: Height function change depends only on neighbouring heights.

- Smoothing: Large valleys are quickly filled.

- Non-linear slope dependence: Vertical effective growth rate does not depend linearly on local slope.

- Space-time independent noise: Growth is driven by noise which quickly decorrelates in space/time and is not heavy-tailed.

It is clear that a proof of the KPZ class solutions behavior for the ballistic deposition model has not been fully investigated mathematically. However, the simulation above suggests that it is true (Fig.2.).

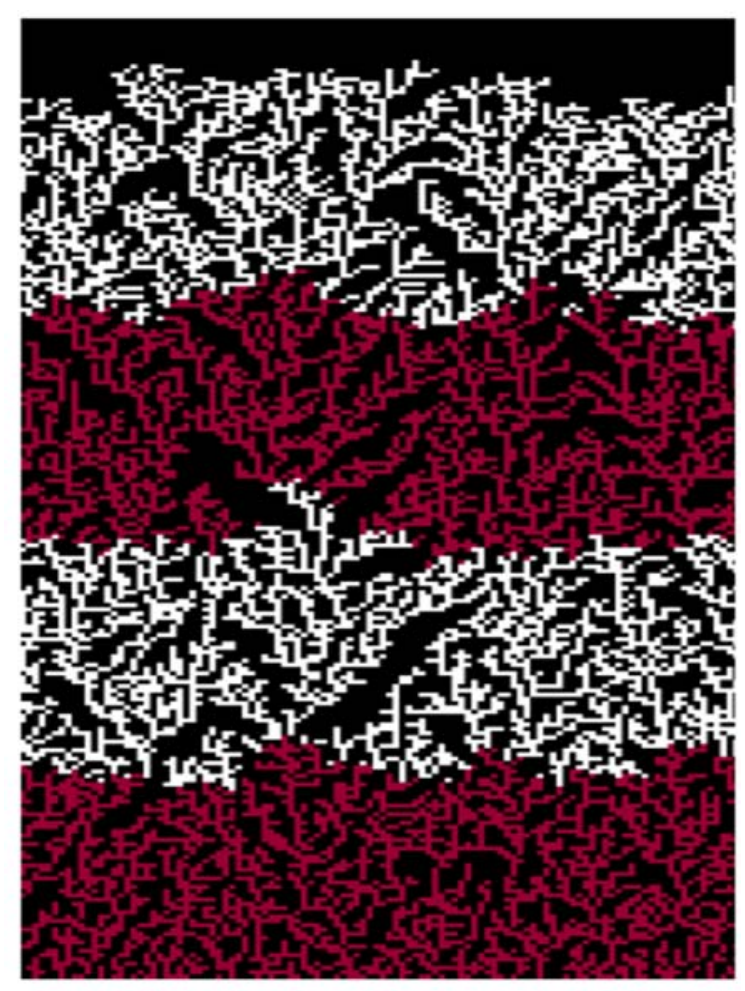

Fig.2. Simulation of ballistic deposition models driven by the same process of falling blocks and run for a long time. The red and white colours represent different epochs of time in the simulation [1].

\section{Are ballistic deposition and the Kardar-Parisi-Zhang equation the same?}

As mentioned above the continuum equation (2.1) which is assumed to give the significant dynamics of the ballistic deposition models is the famous KPZ equation [8], given by

$$
\frac{\partial h}{\partial t}(x, t)=v \nabla^{2} h(x, t)+\frac{\lambda}{2}(\nabla h)^{2}+\eta(x, t)
$$

where $h(x, t)$ is the height of the interface above $x$ at time $t$, and $\eta(x, t)$ is a noise term such that

$$
\eta(x, t)=0 .
$$


Here, we are discussing about connection of formal continuum equation from the ballistic deposition (BD) microscopic rules, that deviates from the KPZ equation. In one dimension these deviations are not important in the presence of noise, but in higher dimensions they are very relevant.

There are currently many suggestions for a direct association between discrete models and continuum equations of motion. There are phenomenological [14] and symmetry [15-18] arguments that can be very illuminating. There is also another approach based on the real-space renormalization-group method [19] which can identify relevant microscopic constants (for example, diffusion) from numerical data when the universality class of the model is already known. Nevertheless, much effort is made to establish a direct relationship between discrete models and continuum equations using formal expansions of discrete equation of motion [2028]. The derivation of the continuum equation is usually based on regularizing and coarse graining discrete Langevin equations which is taken from a Kramers-Moyal expansion of the master equation. It simple means that the transition probabilities are calculated from the microscopic rules of the model for any given discrete height configuration $\left\{h_{i}\right\}$ and includes the discrete $\theta$ (Heaviside) and $\delta$ (Dirac) functions. However, the transition probability is presumably a continuous function, requiring some coarse-graining procedure. In detail, this involves expansions of the form

$$
\theta(x)=1+\sum_{k=1}^{\infty} A_{k} x^{k}
$$

as initially presented in [22]. Also, sometimes less restricted form (2.3) is used

$$
\theta(x)=\sum_{k=0}^{\infty} A_{k} x^{k}
$$

Another suggestion is as follows

$$
\theta(x)=\frac{1+\tanh (C x)}{2}
$$

where $C$ is an arbitrary positive value with exact $\theta(x)$ function as $C \rightarrow \infty$ [25]. Here $C$ is an uncontrolled parameter. In [23], a shifted form is used

$$
\theta(x)=\lim _{C \rightarrow \infty} \frac{1+\tanh (C\{x+\alpha\})}{2}
$$

here $\alpha \in\left(0, \frac{1}{2}\right]$, or using the modified version of $\arctan (C x)$ [24] or $\operatorname{erf}(C x)$ [25] instead of $\tanh (C x)$ in Eq.(2.5). Any version has a number of advantages.

There are some problematic cases with deriving the Kardar-Parisi-Zhang equation [30] from the discrete model known as Ballistic Deposition (BD) [31]. Therefore, a specific case has been developed. The method is based on the discrete Langevin equation. The expansions are used as follows

$$
\theta(x-a)=\theta(x)+\left.\sum_{n=1}^{\infty} \frac{a^{n}}{n_{1}} \frac{\partial^{n} \theta(y)}{\partial y^{n}}\right|_{y=x} .
$$


Also, closely related Langevin based approach is used to represent the max function [28] in order to reach the $\mathrm{KPZ}$ equation from the discrete BD. Recently in [32], using Eq.(2.8), the Edwards-Wilkinson [33] equation was derived from a discrete model using

$$
\theta(x)=\max \{x+a, 0\}=\max \{x\}=\lim _{\varepsilon \rightarrow 0^{+}}\left\{\frac{\varepsilon}{a} \ln \left[\frac{e^{\left(\frac{x+a}{\varepsilon}\right)}+1}{e^{\frac{x}{\varepsilon}}+1}\right]\right\}
$$

where $a$ is any constant in the interval $(0,1]$.

Despite the new and interesting derivation approach, there are three main drawbacks. They are first implemented in one dimension, where the higher dimensions are not discussed at all or cause particularly high difficulties (see, for example, reference [28]).

The second derivation is an expansion like the one given in Eq.(2.3) which is a problem because the Heaviside function is certainly not analytic around zero. Another example is to use an expression such as Eq.(2.5) and expand it for small $C$, while the limiting procedure required to maintain equality requires $C \rightarrow \infty$ [34].

The last is that macroscopic quantities (e.g., the diffusion coefficient) cannot be inferred from microscopic rules with artificial parameters such as $C$ and $\epsilon$, which cannot be removed later.

In the paper [34], it was shown that the absence of formal derivation is not accidental, but rather reflects the significant differences between the continuum equation describing the BD model and the KPZ equation. This difference proves to be slight in one-dimension in the presence of noise, but is crucial when discussing deterministic dynamics. However, there is still an open question: first whether the BD model in $d$ dimensions has a proper continuous description that does not depend on the discrete lattice on which it was defined, and how this equation can be derived. The second is whether the BD model belongs to the KPZ universality class in dimensions higher than one or not.

\section{1+1 dimension study of finite-size scaling of a ballistic deposition model}

In general, the assumption shows that the ballistic deposition model belongs to the KPZ universality class, but it is slow for theoretical predicted values of the exponents $a=\frac{1}{2}, \beta=\frac{1}{3}$, and $z=\frac{3}{2}$ which require more larger lattices simulation. The study [35] used finite scaling and large sampling in order to have rather large lattices with few samples in the simulations. The authors have been focused on the functional size of the various exponents. Finally, only two of the exponents are independent, which we have given above.

In the ballistic deposition model, the interface width is defined by

$$
\langle w(t)\rangle=\sqrt{\left\langle h^{2}(t)\right\rangle-\langle h(t)\rangle^{2}}
$$

where

$$
\left\langle h^{n}(t)\right\rangle=\frac{1}{L}\left\langle\sum_{i=1}^{L} h_{i}^{n}\right\rangle
$$

is the $n$-th moment of the heights at time $t$, and $L$ is the linear dimension of the system. The symbol ' $\langle$ ' represents the average over many realizations.

Thus, the width of the interface follows the scaling function proposed by Family and Vicsek [36], which is defined as: 


$$
w(t)=L^{\alpha} f\left(\frac{t}{L^{z}}\right)
$$

where the scaling function $f(x)$ with $x=t / L^{z}$ is proportional to $f(x)=x^{\beta}$ for $x \ll 1$ and $f(x) \approx$ const. for $x \gg 1$. Here, the exponent $\alpha$ is the roughness exponent with respect to the saturation of the interface width. The exponent $\beta$ is the growth exponent and the exponent $z$ is the crossover or saturation exponent. Since the exponent $z$ represents the transition time from the ballistic deposition regime to the saturation regime, it can characterize finite systems. This is more typical for time-dependent rather than equilibrium systems, where correlations are built up and are eventually reduced by the finite size of the system. The analogue equivalent of equilibrium systems occurs at the critical point of the second order phase transition.

Initially, the interface is growing depending on the size of the lattice and the scaling function of this region is proportional to $t^{\beta} / L^{z \beta}$, we will have the following relation between exponents

$$
\alpha=\beta z
$$

In [35], the simulation of the ballistic deposition model was performed in the square lattice with different lattices of linear sizes $L$, which provided a detailed finite size dependence study. Periodic boundary conditions were applied in a direction perpendicular to the incoming flux of the particles. The ballistic deposition model started to saturate at the instant of time $t_{x}$, which means crossover time. Equation (3.3) showed that the crossover time is consistent with the relation

$$
t_{x} \infty L^{z}
$$

where the symbol ' $\infty$ ' means 'proportional to'. Relation (3.5) initially increases linearly for $t \ll t_{x}$ then takes a constant value.

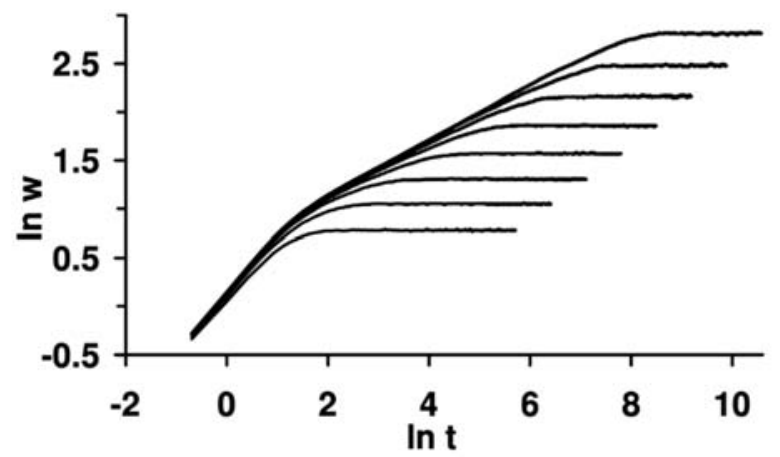

Fig.3. Plot of the width vs. time for systems of sizes $10,20,40,80,160,320,640$, and 1280 lattice constants wide. Smaller sizes saturate at a smaller value of the width [35].

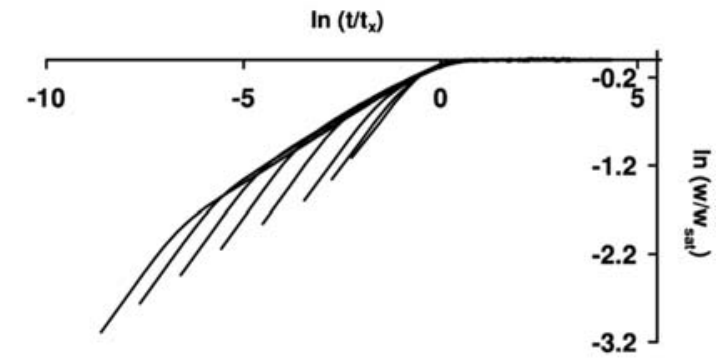

Fig.4. Plot of the collapsed scaling function. Tails represent the initial transient behaviour due to a clean substrate [37].

From Eq.(2.5), the characteristic dependence of the crossover time on the size of the system can be observed as well as the fact that the crossover time increases with the size of the system. The crossover time was defined as the time given by the intersection of the straight line describing the saturation regime and the straight line describing the growth regime. In the process of calculating the slope of the latter straight line, there is a 
transient regime in the initial stages of growth [37]. Figure 4 presents the results for exponent $z$ for the same lattice sizes as in Fig.3. [35].

The value of the exponent $z$ increases monotonically depending on the size of the system. The absolute value of the local slope increases by $\frac{1}{L} \rightarrow 0$ but asymptotic limit of indication has not been reached yet. Since the exponent $\beta$ characterizes the growth of the interface for finite system size in early times, we can understand from [35] that smaller lattice constants lead to a non-monotonic approach to the asymptotic value. Although the conservative value $\beta \geq 0.30$ can be obtained by extrapolation, it is not clear whether it can converge to the value 0.3 [38] predicted by the KPZ universality class. Later the system had time to grow lateral correlations much longer than its own linear size, but since the spatial correlations are limited by the size of the system, it showed that the system has a natural length scale, the saturation width of the interface. Because of Eq.(3.3) and Fig.3, we expect the value of the saturated width of the interface to scale as $L^{\alpha}$. As with previous quantities the roughness exponent $\alpha$ did not saturate. It is also instructive to observe the value of the exponent $\alpha$ given from Eq.(3.4). The value of $\alpha$ is taken from $\beta z$ for a given size of the system. Again, the results do not converge to the asymptotic values. Finally, the values of the $\mathrm{z}$ and exponent $\alpha$ have also to satisfy the following scaling relation

$$
z+\alpha=2,
$$

thus, this quantity is plotted for various system sizes. Again, we can observe that the sum of two values is far from being achieved through the trend in the right direction. (See Figure 6).

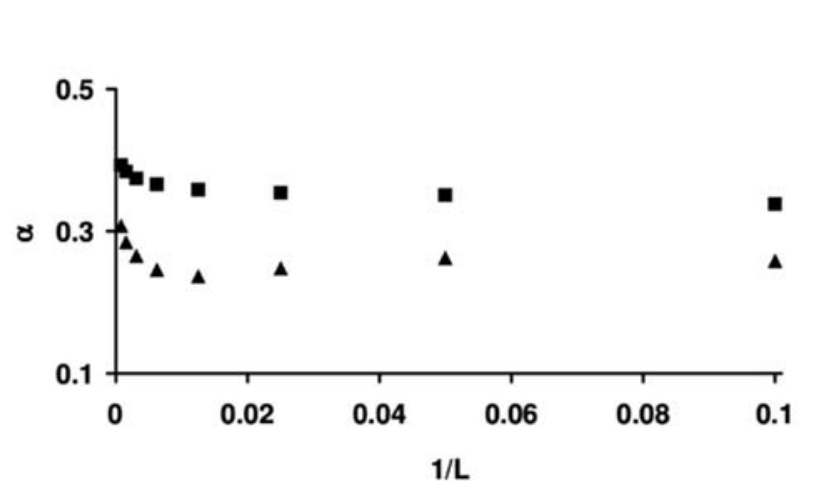

Fig.5. Plot of the roughness exponent, $\alpha$, vs. 1/L for the same system sizes referred to in Figure 3. Filled triangles are calculated using Eq.(14), while filled rectangles are the values directly calculated [35].

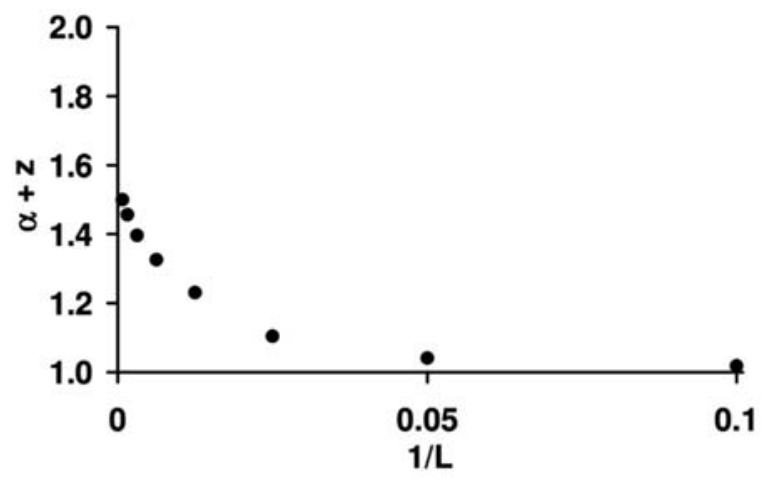

Fig.6. The plot shows the quantity $\alpha+z$ vs. $1 / L$. Refer to the text for further details [35].

The ballistic deposition model shows strong corrections to scaling up to lattice sizes of 1280 lattice constants wide, leading to quite low convergence rates towards the asymptotic values of the exponents $\alpha, \beta$, and $\mathrm{z}$. Results presented in [35] indicate that correction scale higher than 1280 has not been investigated and it requires more processes of simulating larger lattices to obtain better estimates.

$1+1$ dimension study of finite-size scaling of the ballistic deposition model provides an example of how nanostructured materials may introduce more stringent demands on present day theories. In particular, the slow convergence of the exponents $\alpha, \beta$, and $\mathrm{z}$ to their asymptotic limit may prevent some real systems from being in the asymptotic regime. However, it requires more study on the KPZ universality model and its connection with the ballistic deposition model. 


\section{Direct connection between the BD and the KPZ equation using a limiting procedure}

Takashi Nagatani [45] described a direct and formal derivation of the KPZ equation from the ballistic deposition models. Figure 1 shows that the particle sticks to the first site along its trajectory and occupies nearest neighbour. It represents ballistic deposition as $i-1, i, i+1$. At time $t+1$, the height $h(i, t+1)$ is given by

$$
h(i, t+1)=\max [h(i-1, t), h(i, t)+1, h(i+1, t)]
$$

where $\max []$ is the maximum function. In [45] a limiting procedure was applied to the difference of heights between nearest neighbours and the following Eq.(4.2) was obtained

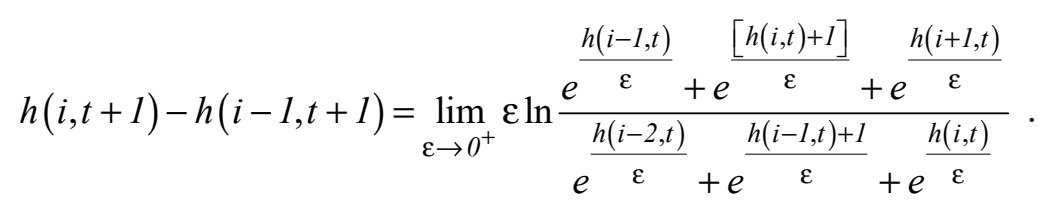

When $e^{\frac{h(i, t)-h(i-1, t)}{\varepsilon}}$ is replaced by $c(i, t)$ a difference-difference equation was obtained

$$
\begin{aligned}
& c(i, t+1)=[\delta c(i-1, t)+c(i-1, t) c(i, t)+ \\
& +\delta c(i-1, t) c(i, t) c(i+1, t)][\delta+c(i-1, t)+\delta c(i-1, t) c(i, t)]^{-1}
\end{aligned}
$$

where $\delta=e^{-\frac{1}{\varepsilon}}$. We consider that the hydrodynamic mode on in the rough surface in the coarse-grained scales. The perturbation method applied to Eq.(4.3) defined slow variables $X$ and $T$ for the space variables $i$ and time variables $t$ [46].

For $|\Delta x| \ll 1$, we get $X=(\Delta x) i, T=\delta(\Delta x)^{2} t$. By setting

$$
\ln c(i, t)=(\Delta x) v\left(\Delta x i, \delta(\Delta x)^{2} t\right)=(\Delta x) v(X, T)
$$

and expanding $c(i, t)$ to order $(\Delta x)^{3}$, the following formula was obtained

$$
c(i, t)=\exp [(\Delta x) v(X, T)]=1+(\Delta x) v+\frac{(\Delta x)^{2} v^{2}}{2}+\frac{(\Delta x)^{3} v^{3}}{6}+\ldots
$$

where $v$ equals $v(X, T)$ in the second equality. Similarly, $c(i-1, t), c(i+1, t)$ and $c(i, t+1)$ were expanded in [45] and by substituting long-wavelength expansion into Eq.(2.4) keeping only the third term, the Burgers equation was obtained

$$
\partial_{T} v=\left(2 v \partial_{X} v+\partial_{X}^{2} v\right)(1+2 \delta)
$$


By setting $v(X, T)=\frac{h(i, t)-h(i-1, t)}{\varepsilon \Delta x}=\partial_{X} h$, the KPZ equation is obtained

$$
\partial_{T} h=\frac{\left(\partial_{X} h\right)^{2}+\partial_{X}^{2} h}{1+2 \delta}
$$

Here $\delta$ constant is given by the ratio of the time increment $\Delta t$ to the square of the space increment $\Delta x$. The ballistic deposition process on coarse-grained scales can be described by the KPZ equation.

In addition, there was an attempt at modifying models for the ballistic deposition with next nearest neighbour sticking rule in the same sequence as Eqs (4.1)-(4.4). However, the application of the limiting procedure and the perturbation method to the (2+1)-dimensional ballistic deposition could not derive the KPZ equation.

\section{Conclusion}

It is always believed [38] that ballistic deposition is a paradigmatic discrete model for the KPZ universality class, but a formal derivation has not been obtained. In this work, we analysed that the absence of a formal derivation is not accidental. It rather showed important differences between the continuum equation that describes the ballistic deposition model and the KPZ equation. The presence and effect of noise were low, but it was significant for the deterministic dynamics in one-dimension [47]. In addition, the ballistic deposition model showed strong corrections to scaling up to lattice sizes of 1280 lattice constants wide, which led to quite low convergence rates towards the asymptotic values of the exponents $\alpha, \beta$, and $\mathrm{z}$.

Both the limiting procedure and perturbation methods for the derivation of the KPZ equation from the ballistic deposition models were presented. A strong connection between the ballistic deposition and the KPZ equation was shown. Finally, in spite of successful derivation of the KPZ equation in $1+1$ dimensions from the ballistic deposition, $2+1$ dimension did not show expected results.

\section{Acknowledgment}

This work was supported by Project No. 129257 implemented with the support provided from the National Research, Development and Innovation Fund of Hungary, financed under the K_18 funding scheme.

\section{Nomenclature}

$$
\begin{aligned}
C & - \text { arbitrary positive value } \\
c_{1}, c_{2}, a, \beta, z & - \text { constant numbers } \\
h(t, x) & - \text { height function } \\
h_{i} & \text { - height configuration } \\
i & - \text { space variable } \\
L & - \text { linear dimension } \\
t & \text { - time variable } \\
t_{x} & - \text { crossover time } \\
w(t) & - \text { interface width } \\
X, T & - \text { slow variables } \\
x, y, z & - \text { coordinate system } \mathrm{x} \\
\eta & - \text { noise } \\
\delta & - \text { Dirac function } \\
\theta & - \text { Heaviside discrete }
\end{aligned}
$$




\section{References}

[1] Tiller W.A. (1991): The Science of Crystallization, Macroscopic.- Phenomena and Defect Generation Cambridge University Press, Cambridge, UK.

[2] Ritter G.C., Matthai O., Takai A., Rocher A., Cullis S., Ranganathan S. and Kuroda K., Eds. (1998): Recent Developments in Thin Film Research: Epitaxial Growth and Nanostructures, Electron Microscopy and X-Ray Diffraction. Elsevier Science.- Amsterdam.

[3] G. Vekilov G., Iwan J. and Alexander D. (2000): Dynamics of layer growth in protein crystallization.- Chemical Reviews 100, No. 6., pp.2061-2090.

[4] Jackson K.A. (2006): Kinetic Processes: Crystal Growth, Diffusion, and Phase Transformations in Materials.- John Wiley \& Sons.

[5] Chernov A.A. and Nishinaga T. (1987): Growth shapes and their stability at anisotropic interface kinetics: theoretical aspects for solution growth.- Morphology of Crystals, Terra Science Publishers, Tokyo, pp.207-267.

[6] Saito Y. (1996): Statistical physics of crystal growth.- World Scientific.

[7] Pimpinelli A. and Villain J. (1999): Physics of crystal growth.- Cambridge University Press, p.400.

[8] Michely T. and Krug J. (2004): Mounds Islands. Atoms.- Springer Series in Surface Science, vol.42, SpringerVerlag Berlin Heidelberg, 10.1007/978-3-642-18672-1.

[9] Aqua J-N. (2013): Growth and self-organization of SiGe nanostructures.- Physics Reports, vol.522, No.2, pp.59-189.

[10] Vold M.J. (1959): A numerical approach to the problem of sediment volume.- J. Colloid Sci., vo.14, No.2, pp.168-174.

[11] Corwin I. (2016) Kardar-Parisi-Zhang universality.- Notices of the AMS, vol.63, No.3, pp.230-239.

[12] Tracy C. and Widom H. (1994): Level-spacing distributions and the Airy kernel.- Commun. Math. Phys., vol.159, pp.151-174.

[13] Tracy C. and Widom H. (1996): On orthogonal and symplectic matrix ensembles.- Commun. Math. Phys., vol.177, pp.727-754.

[14] Villain J. (1991): Continuum models of crystal growth from atomic beams with and without desorption.- Journal de Physique, vol.1, No.1, pp.19-42.

[15] Barabasi A.-L. and Stanley E. (1995): Fractal Concepts in Surface Growth.- Cambridge Univ. Press, Cambridge.

[16] Kardar M., Parisi G. and Zhang Y.C. (1986): Dynamic scaling of growing interfaces.- Physical Review Letters, vol.56, No.9, p.889-892.

[17] Hwa T. and Kardar M. (1992): Avalanches, hydrodynamics, and discharge events in models of sandpiles.- Physical Review A, vol.45, No.10, p.7002-7023.

[18] Marsili M., Maritan A., Toigo F. and Banavar J.R. (1996): Stochastic growth equations and reparametrization invariance.- Reviews of Modern Physics, vol.68, No.4, pp.963-983.

[19] Lam C.-H. and Sander L.M. (1993): Inverse method for interface problems.- Physical Review Letters, vol.71, No.4, 561-564.

[20] Park S.-C., Kim D. and ParkJ.-M. (2001): Derivation of continuum stochastic equations for discrete growth models.Physical Review E, vol.65, No.1, pp.1-4, https://doi.org/10.1103/PhysRevE.65.015102.

[21] Racz Z., Siegert M., Liu D. and Plischke M. (1991): Scaling properties of driven interfaces: symmetries, conservation laws, and the role of constraints.- Physical Review A, vol.43, No.10, pp.5275-5283.

[22] Vvedensky D.D., Zangwill A., Luse C.N. and Wilby M.R. (1993): Stochastic equations of motion for epitaxial growth.- Physical Review E, vol.48, No.2, pp.852-862.

[23] Predota M. and Kotrla M. (1996): Stochastic equations for simple discrete models of epitaxial growth.- Physical Review E, vol.54, No.4, pp.3933-3942.

[24] Costanza G. (1997): Langevin equations and surface growth.- Physical Review E 55, No.6, pp.6501-6506.

[25] Bantay P. and Janosi I.M. (1992): Avalanche dynamics from anomalous diffusion.- Physical Review Letters, vol.68, No.13, pp.2058-2061.

[26] Corral A. and Diaz-Guilera A. (1997): Symmetries and fixed point stability of stochastic differential equations modeling self-organized criticality.- Physical Review E 55, No.3, pp.2434-2445.

[27] Tokihiro T., Takahashi D., Matsukidaira J. and Satsuma J. (1996): From soliton equations to integrable cellular automata through a limiting procedure.- Physical Review Letters, vol.76, No.18, pp.3247-3250.

[28] Nagatani T. (1998): From ballistic deposition to the Kardar-Parisi-Zhang equation through a limiting procedure.Physical Review E, vol.58, No.1, pp.700-703.

[29] Vvedensky D.D. (2003): Edwards-Wilkinson equation from lattice transition rules.- Physical Review E, vol.67, No.2, pp.1-4.

[30] Kardar M., Parisi G., Zhang Y.-C. (1986): Dynamic scaling of growing interfaces.- Physical Review Letters, vol.56, No.9, pp.889-892. 
[31] Costanza G. (1997): Langevin equations and surface growth.- Physical Review E, vol.55, No.6, pp.6501-6506.

[32] Vvedensky D.D. (2003): Edwards-Wilkinson equation from lattice transition rules.- Physical Review E, vol.67, No.2, pp.1-4.

[33] Edwards S.F. and Wilkinson D.R. (1982): The surface statistics of a granular aggregate.- Proceedings of the Royal Society of London. A. Mathematical and Physical Sciences, vol.381, No.1780, pp.17-31.

[34] Katzav E. and Schwartz M. (2004): What is the connection between ballistic deposition and the Kardar-ParisiZhang equation?- Physical Review E, vol.70, No.6, pp.1-8.

[35] Miranda R., Ramos M. and Cadilhe A. (2003): Finite-size scaling study of the ballistic deposition model in (1+ 1)dimensions.- Computational Materials Science, vol.27, No.1-2, pp.224-229.

[36] Family F. and Vicsek T. (1985): Scaling of the active zone in the Eden process on percolation networks and the ballistic deposition model.- Journal of Physics A: Mathematical and General, vol.18, No.2, p.75.

[37] Cadilhe A.M., Stoldt C.R., Jenks C.J., Thiel P.A. and Evans J.W. (2000): Evolution of far-from-equilibrium nanostructures on Ag (100) surfaces: Protrusions and indentations at extended step edges.- Physical Review B, vol.61, No.7, pp.4910-4925.

[38] Family F. and Vicsek T. (1991): Dynamics of Fractal Surfaces.- World Scientific.

[39] Corwin I. (2016): Kardar-Parisi-Zhang universality.- Notices of the AMS, vol.63, No.3, pp.230-239.

[40] Bognár G. (2020): Roughening in nonlinear surface growth model.- Applied Sciences, vol.10, No.4, p.10. https://doi.org/10.3390/app10041422

[41] Barna I.F., Bognár G., Guedda M., Mátyás L. and Hriczó K. (2020): Analytic self-similar solutions of the KardarParisi-Zhang interface growing equation with various noise terms.- Mathematical Modelling and Analysis, vol.25, No.2, pp.241-256. 2020. https://doi.org/10.3846/mma.2020.10459.

[42] Barna I.F., Bognár G., Guedda M., Mátyás L. and Hriczó K. (2019): Analytic traveling-wave solutions of the KardarParisi-Zhang interface growing equation with different kind of noise terms.- In book: Differential and Difference Equations with Applications, ICDDEA, Lisbon, Portugal, pp.239-253, arXiv:1908.09615.

[43] Sayfidinov O. and Bognár G. (2020): Numerical solutions of the Kardar-Parisi-Zhang interface growing equation with different noise terms.- In: Jármai K., Voith K. (eds) Vehicle and Automotive Engineering 3. VAE 2020. Lecture Notes in Mechanical Engineering. Springer, vol.3, pp.302-311, https://doi.org/10.1007/978-981-159529-5_27.

[44] Bognár G. (2020): Roughening in nonlinear surface growth model.- Applied Sciences, vol.10, No.4, p.1422, doi:10.3390/app10041422.

[45] Nagatani T. (1998): From ballistic deposition to the Kardar-Parisi-Zhang equation through a limiting procedure.Physical Review E, vol.58, No.1, pp.700-703.

[46] Cross M.C. and Hohenberg P.C. (1993): Pattern formation outside of equilibrium.- Reviews of Modern Physics, vol.65, No.3, pp.851-1112.

[47] Sayfidinov O. and Bognár G. (2020): One dimensional Kardar-Parisi-Zhang equation in various initial condition amplitudes.- Journal of Advances in Applied \& Computational Mathematics, vol.7, pp.32-37.

Received: April 22, 2021

Revised: October 4, 2021 